\title{
Etude des interactions entre constituants du lait et membranes minérales de microfiltration
}

\author{
Claudine VETIER *, M. BENNASAR * et B. TARODO DE LA FUENTE * \\ avec la collaboration technique de G. NABIAS** \\ * Laboratoire de Technologie alimentaire \\ Groupe de Recherche et de Développement sur les Membranes \\ Université des Sciences et Techniques du Languedoc \\ place E. Bataillon - 34060 Montpellier cedex, France \\ ** Laboratoire de Chimie des Matériaux (C.N.R.S. - L.A.314) \\ Groupe de Recherche et de Développement sur les Membranes \\ Ecole Nationale Supérieure de Chimie \\ 8, rue de l'Ecole-Normale - 34075 Montpellier cedex, France
}

\section{Résumé}

Cette étude n'est qu'une étape dans la compréhension des phénomènes de polarisation de concentration et de colmatage lors de la micro- ou de l'ultrafiltration du lait sur membrane minérale. Elle consiste en la mise au point d'une méthode couplant la microscopie électronique à balayage et l'analyse biochimique des colmatages obtenus au repos (sans l'influence des facteurs vitesse et pression). Elle a permis de mettre en évidence l'existence d'un colmatage différencié dans lequel on a pu distinguer :

- une adsorption initiale des constituants non micellaires sur les grains d'alumine membranaire ;

— un colmatage peu profond à l'intérieur de la membrane ;

- un dépôt en surface quantitativement important (60 à $80 \%$ du colmatage total) caractérisé par une structure poreuse constituée par l'agglomération des micelles de caséines et contenant des globules gras dans le cas d'un lait entier.

Nous avons confirmé sa nature essentiellement protéique avec présence de tous les constituants d'origine du lait et la participation importante des sels phosphocalciques. La présence de la matière grasse semble jouer un rôle important puisque l'on a constaté une évolution différente au cours du temps de contact suivant que le lait est entier (augmentation du dépôt) ou écrémé (diminution du dépôt).

Mots clés : Microfiltration - Ultrafiltration - Membranes - Colmatage - Lait - Microscopie électronique à balayage.

\section{Summary}

\section{Milk mineral membrane interactions}

This study is only a stage in the understanding of the phenomena of polarisation concentration and fouling during micro or ultrafiltration of milk on mineral membrane. 
It uses scanning electron microscopy and biochemical analysis of fouling obtained in static conditions (without the effect of flow velocity and pressure). This method reveals fouling in which we have distinguished :

- an initial adsorption of non-micellar constituents on the alumina grain of the membrane,

- shallow fouling inside the membrane,

- a considerable amount of surface deposit (60 to $80 \%$ of total fouling) with a porous structure: aggregation of casein micelles with fat globules in the case of whole milk. We have confirmed proteic nature of fouling with the presence of all the milk constituents and the important contribution of salts such as calcium and phosphorus. Fat also seems to play an important part in the evolution of fouling.

Key words : Microfiltration - Ultrafiltration - Membranes - Fouling - Milk - Scanning Electron Microscopy.

\section{Introduction}

Lors d'une filtration tangentielle sur membrane, le principal facteur limitant le débit de perméation est la formation de la couche de polarisation qui conduit au colmatage de la membrane et on peut rappeler quelques-uns des travaux déjà effectués dans ce domaine :

PORTER en 1972 a étudié la polarisation de concentration avec les membranes d'ultrafiltration. En 1975, LEE et al. ont réalisé, par microscopie électronique à balayage, l'examen des protéines solubles du lactosérum et ont fait le lien avec le colmatage des membranes lors de l'ultrafiltration.

En 1980, Gernedel publie ses travaux relatifs à l'ultrafiltration du lait et aux facteurs influençant l'établissement de la couche de colmatage. Plus récemment, BAKLOUTi et al. (1984) ont étudié l'adsorption et le transfert de matière en ultrafiltration. Enfin nous-même avons mis en évidence l'existence de ces phénomènes lors de la microfiltration du vin sur membrane minérale (POIRIER, 1985).

L'étude dynamique de ces phénomènes étant difficile, nous avons choisi une approche différente de tout ce qui a été fait jusqu'à présent en réalisant une étude statique, donc volontairement simplifiée, du colmatage obtenu sur une membrane minérale de microfiltration utilisée pour l'ultrafiltration du lait (BENNASAR, 1984). Nous rapportons donc la mise au point d'une méthode d'analyse pour identifier la nature et la structure du colmatage qui se forme au repos dès que la solution à traiter et la membrane sont mises en contact. Dans ces conditions, l'influence des paramètres vitesse et pression est supprimée et seuls interviennent les phénomènes de colmatage mécanique et d'adsorption des constituants du lait sur le matériau membranaire.

Nous avons fait appel en parallèle à l'observation directe du colmatage par microscopie électronique à balayage, technique de plus en plus utilisée dans l'étude des produits alimentaires et en particulier des produits laitiers (GLOVER et BrooKER, 1974 ; KALAB, 1981) et à l'analyse biochimique du dépôt remis en solution dans des conditions données. 


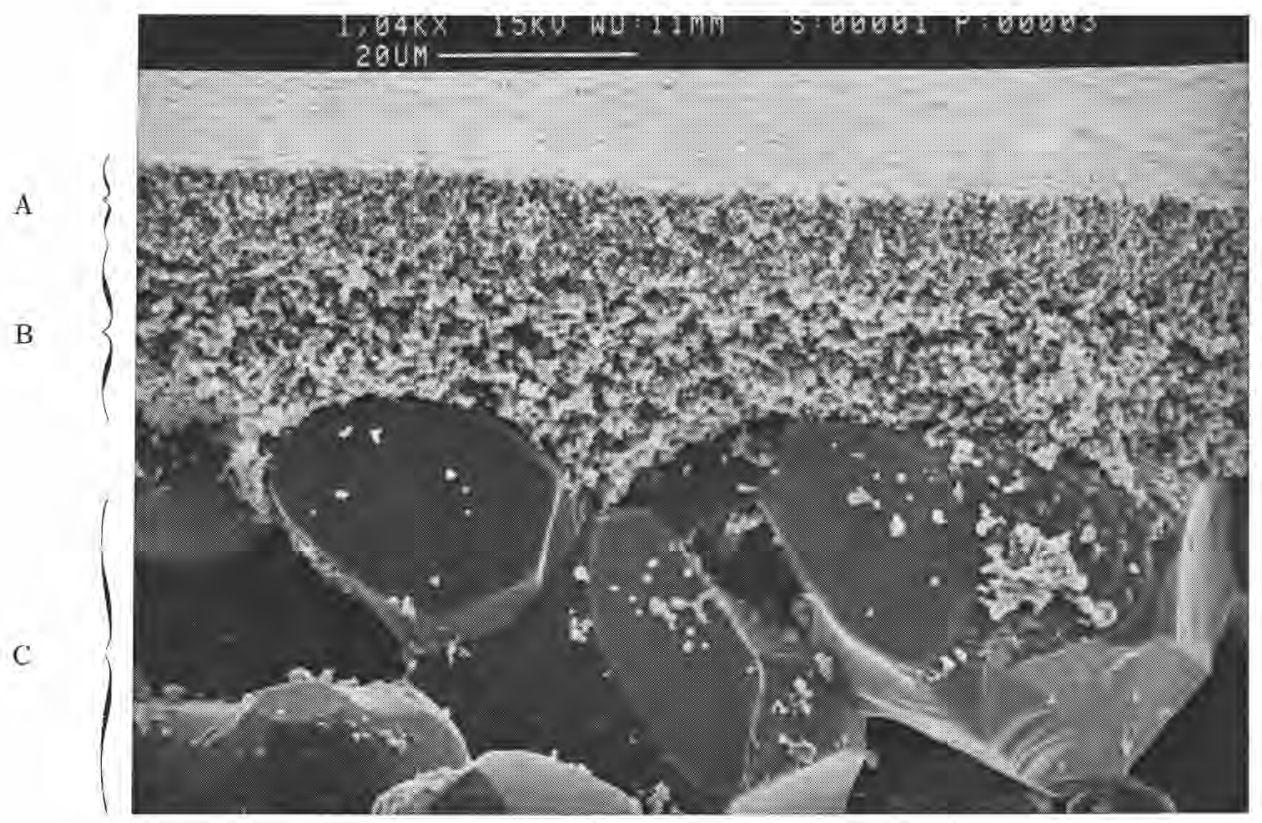

Fig. 1 a

Membrane Ceraver en alumine: coupe avec trois couches caractéristiques:

A. En surface, la couche filtrante de diamètre moyen de pores $0,2 \mu m$ et d'épaisseur environ $10 \mu \mathrm{m}$.

$B$. Sous-couche filtrante de diamètre moyen de pores $0,8 \mu \mathrm{m}$ et d'épaisseur environ $15 \mu \mathrm{m}$.

C. Support poreux de diamètre moyen de pores $10 \mu \mathrm{m}$ et d'épaisseur environ $2 \mathrm{~mm}$.

Ceraver membrane of alumina ; section with three characteristic layers :

A. At surface: filter layer with pores average diameter of $0,2 \mu \mathrm{m}$ and thickness of $10 \mu \mathrm{m}$.

B. Under-filter layer with pores average diameter of $0,8 \mu \mathrm{m}$ and thickness of $15 \mu \mathrm{m}$.

C. Porous support with pores average diameter of $10 \mu \mathrm{m}$ and thickness of $2 \mathrm{~mm}$.

\section{Matériel et méthodes}

- Membrane minérale Ceraver. Couche filtrante et support en alumine (fig, 1 a et b). Tronçon tubulaire de $7,5 \mathrm{~cm}$ de long et de diamètre interne $1,5 \mathrm{~cm}$, soit une surface de $3,3 \cdot 10^{-3} \mathrm{~m}^{2}$. Diamètre moyen des pores : $0,2 \mu \mathrm{m}$.

- Laits. Différents types de lait sont utilisés :

- lait écrémé France-Lait (qualité sans antibiotique) reconstitué à $10 \%$ $(\mathrm{M} / \mathrm{V})$ dans de l'eau permutée et stocké à $+4^{\circ} \mathrm{C}$ pendant $12 \mathrm{~h}$;

- lait cru de mélange entier et écrémé.

- Colmatage de la membrane (fig. $2 \mathrm{a}$ et $2 \mathrm{~b}$ ). Obtenu par contact entre la membrane et le lait pendant une durée variable $(5 \mathrm{~min}, 30 \mathrm{~min} ; 1,2$ et $5 \mathrm{~h})$ à température ambiante. Le tube est maintenu plein pendant toute la durée de l'essai par apport continu de lait destiné destiné à compenser la perméation due à la 


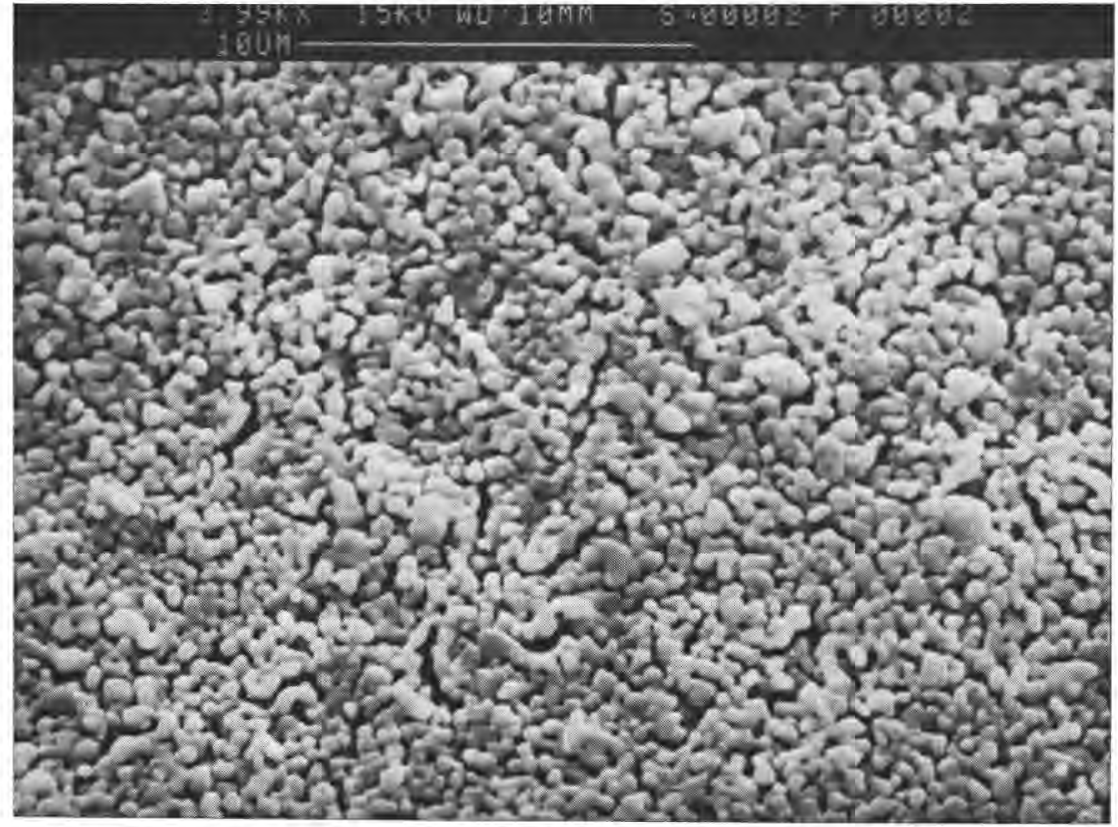

Fig. $1 \mathrm{~b}$

Membrane Ceraver : surface de la couche filtrante constituée par la juxtaposition des grains d'alumine - diamètre moyen de pores $0,2 \mu \mathrm{m}$.

Ceraver membrane: filter layer surface made of alumina grains "side by side putting " - pores average diameter : $0,2 \mu \mathrm{m}$.

pression hydrostatique et aux forces de capillarité. En fin d'essai le lait est éliminé par vidange du tube et le dépôt fixé sur la membrane sera analysé. Compte tenu des vitesses de perméations variables suivant le type de lait et des temps de contact différents, les volumes de lait mis en œuvre sont compris entre 12 et $16 \mathrm{ml}$.

- Récupération du colmatage et mise en solution. La membrane avec son dépôt est rincée avec agitation dans $140 \mathrm{ml}$ d'eau permutée pendant $30 \mathrm{~s}$, puis égouttée $15 \mathrm{~s}$ au-dessus de ce bain; cette première solution de récupération est appelée RAA. La récupération du dépôt proprement dit est effectuée au moyen d'ultrasons (cuve Eurosonic 22) par trempage durant $15 \mathrm{~min}$ chaque fois dans 3 bains successifs d'eau permutée $(30 \mathrm{ml})$. La solution globale correspondante est appelée US. Les solutions RAA et US sont ensuite analysées : matières azotées totales, calcium, phosphore, électrophorèse.

Après ces divers traitements la membrane est broyée grossièrement dans un mortier avant d'être soumise à l'analyse (matières azotées totales), cette dernière fraction est nommée AM.

- Observation en microscopie électronique à balayage (MEB). Réalisée sur des échantillons de membrane colmatée de surface inférieure ou égale à $1 \mathrm{~cm}^{2}$ 


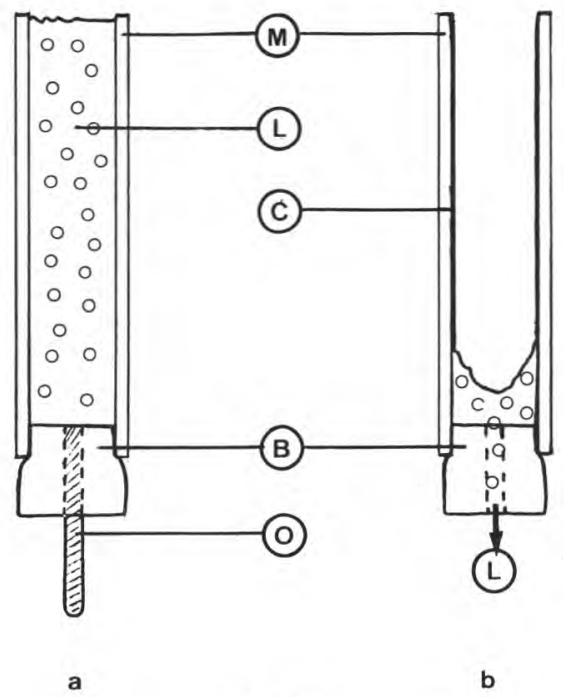

Fig. 2

$a:$ Contact lait-membrane

$b:$ Elimination du lait

$M$ : Membrane minérale.

$L$ : Lait.

$C$ : Colmatage laissé par le lait sur la membrane.

$B$ : Bouchon.

$O$ : Obturateur.

a: Milk membrane contact

$b:$ Milk elimination

$M$ : Mineral membrane.

$L$ : Milk.

$C$ : Fouling.

$B$ : Cap.

$O$ : Shutter.

prélevés dans la partie médiane du tube. Pour des raisons techniques (pollution du milieu poreux membranaire par les eaux de rinçage), les échantillons ne subissent pas de rinçage avant l'analyse mais seulement un égouttage. L'importance du dépôt observé est de ce fait surestimée mais seulement au niveau de la couche de polarisation (solution de récupération RAA).

Nous avons utilisé successivement trois techniques de fixation de ces échantillons :

a) Séchage à l'air à température ambiante (faible grossissement).

b) Congélation rapide dans les vapeurs d'azote liquide à $-100^{\circ} \mathrm{C}$ en $20 \mathrm{~min}$ environ suivie immédiatement d'une lyophilisation à $-60^{\circ} \mathrm{C}$.

c) Fixation chimique par trempage dans une solution de glutaraldéhyde à $2,5 \%$ en tampon phosphate $\mathrm{pH} 7,00$ pendant $30 \mathrm{~min}$ à température ambiante ou par maintien dans les vapeurs de glutaraldéhyde à $1 \%$ pendant $6 \mathrm{~h}$. Puis congélation et lyophilisation suivant la technique précédemment décrite $(b)$ (CARroll et al., 1968 ; Lee et al., 1975 ; Kalab, 1981, 1984). 
Les échantillons ainsi fixés sont ensuite montés sur les porte-objets et métallisés à l'or (Polaron E 5000) puis observés grâce à un MEB Cambridge Stereoscan 100 à des tensions d'accélération de 10 à $25 \mathrm{KV}$.

- Analyses biochimiques :

- dosage des matières azotées totales (MAT) par la méthode de Kjehldahl avec un appareil Tecator Kjeltec ;

- dosage de l'ion calcium (Ca) par spectrophotométrie d'absorption atomique avec un appareil Varian Techtron AA6 ;

- dosage du phosphore $(\mathrm{P})$ par la méthode colorimétrique au phosphomolybdate d'ammonium; normes internationales de 1a FIL $\mathrm{n}^{\circ} 42$ (PIEN, 1969); spectrophotomètre Bausch et Lomb Spectronic 2000;

- électrophorèse en gel de polyacrylamide sur appareil Camag selon la méthode préconisée par le fabricant du matériel (4132 Muttenz, Suisse, Sonnenmattstrasse-11), en milieu urée 5M.

\section{Résultats et discussions}

\section{A. Description et observation du colmatage}

Cette première partie a pour objectif de confronter les hypothèses formulées dans nos précédents travaux sur l'ultrafiltration du lait (BENNASAR, 1984), concernant le mécanisme de formation du colmatage avec nos premières observations en microscopie électronique à balayage des dépôts obtenus en statique. Rappelons que BENNASAR (1984) décrit trois phénomènes distincts au voisinage de la membrane (fig. 3) :

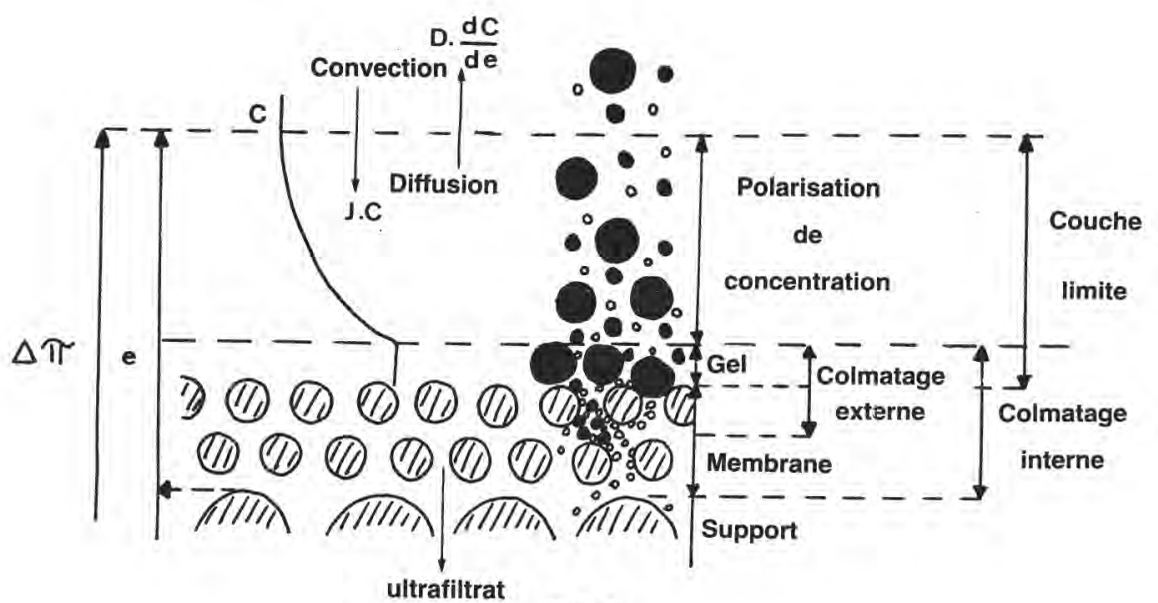

Fig. 3

Schéma de principe de l'ultrafiltration sur membrane minérale (selon BENNASAR, 1984) appliquée à notre étude statique (en hachuré : l'alumine).

Diagram of mineral membrane ultrafiltration theory (according to BENNASAR, 1984) adapting at our static study (in hatching : alumina grain). 


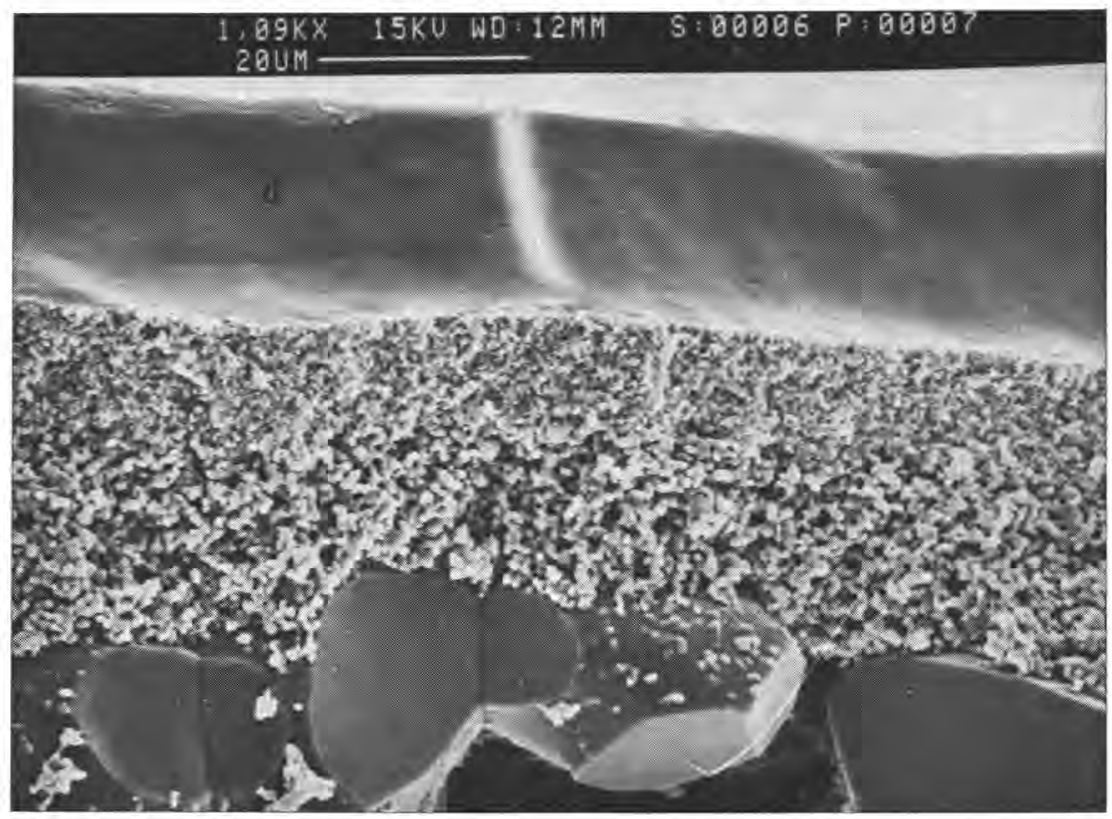

Fig. 4 a

Membrane colmatée après 30 min de contact avec le lait écrémé reconstitué (fixation a) $d=$ dépôt des constituants du lait (épaisseur $=20 \mu \mathrm{m}$ ).

Membrane fouling after skimmilk contact time of $30 \mathrm{~min}$ (fixation a) $d=$ deposit of milk constituents (Thickness $=20 \mu \mathrm{m}$ ) .

- la polarisation de concentration qui conduit à une zone de concentration élevée des solutés à l'intérieur de la couche limite hydrodynamique. Si cette concentration est suffisante, elle peut induire la formation d'un dépôt plus ou moins structuré en surface de la membrane;

- le colmatage externe initial dû à une rétention mécanique et (ou) une adsorption physico-chimique immédiate des particules et des molécules en surface de la membrane et dans les couches adjacentes des grains d'alumine ;

- le colmatage interne progressif qui résulte de la pénétration et de la rétention mécanique ou physico-chimique en fonction du temps de petites particules et molécules à l'intérieur du réseau filtrant (dépôt et membrane).

La figure $4 \mathrm{a}$ permet à un faible grossissement de visualiser l'ensemble du dépôt en surface de la membrane. adsorbé.

La figure $4 \mathrm{~b}$ montre la présence d'un colmatage différencié : dépôt et film

La structure fine du dépôt constitué essentiellement de micelles de caséine, comme le montrent les figures $4 \mathrm{c}$ et $4 \mathrm{~d}$ (surface et coupe), est bien celle d'un lit poreux identique à la membrane, mais composé d'éléments de taille beaucoup plus faible. C'est un milieu poreux semblable qui avait été pris comme modèle par KoZENY (1927) et CARMAN $(1937,1939)$ pour établir les lois de la filtration puis par PorTer et Michaels (1972) pour celles de l'ultrafiltration et c'est l'hypothèse 


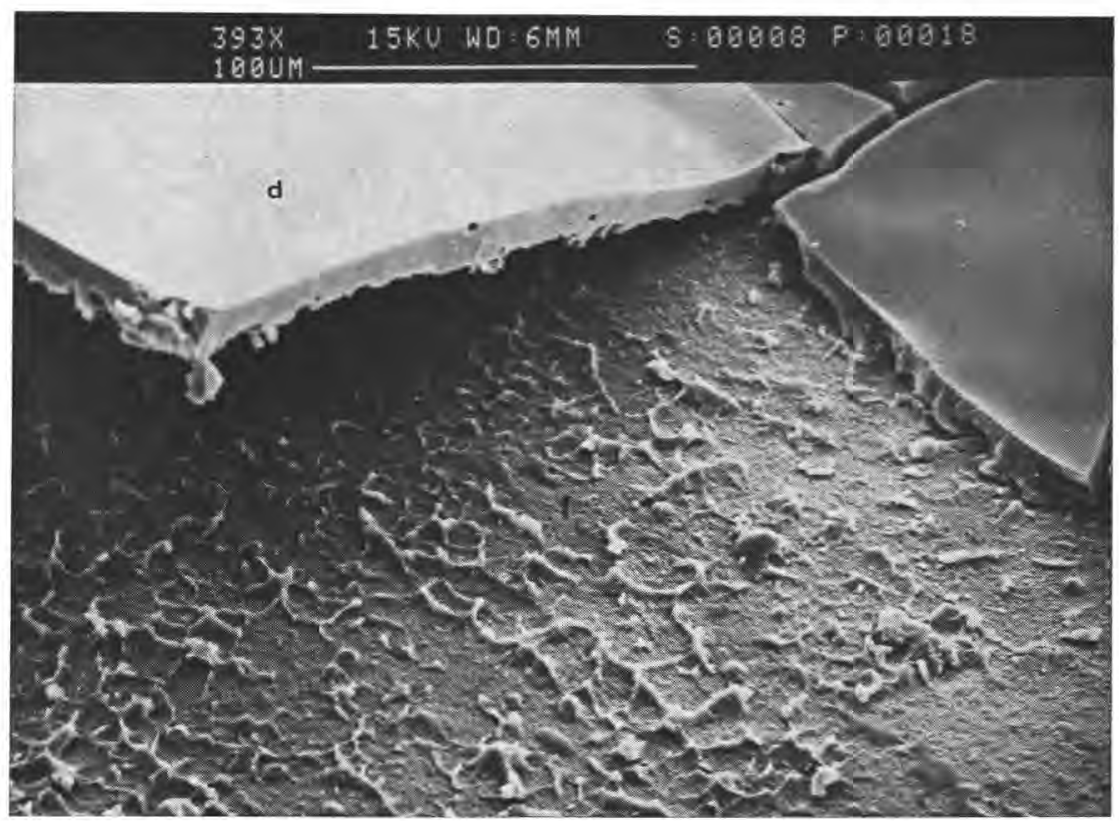

Fig. $4 \mathrm{~b}$

Membrane colmatée après $5 \mathrm{~h}$ de contact avec le lait écrémé (fixation b). Dépôt (d) en partie décollé de la membrane laissant apercevoir un film $(f)$ adsorbé sur les grains Membrane fouling after raw skimmilk contact time of 5 hours (fixation b). Deposit (d) and adsorbed film ( $f$ ) on the alumina grains.

que nous avions nous-mêmes formulée plus récemment (BENNASAR, 1984) qui se trouve ainsi vérifiée.

La figure $4 \mathrm{e}$ montre que l'adsorption initiale et le colmatage interne n'affectent la membrane que sur une très faible épaisseur : seuls les grains d'alumine des premières couches sont enrobés et quelquefois soudés entre eux et les micelles ne pénètrent que sur 1 ou $2 \mu \mathrm{m}$ d'épaisseur dans la membrane.

\section{B. Influence de l'état de surface de l'alumine sur le colmatage en fonction du temps (tabl. 1)}

Les travaux déjà effectués dans notre laboratoire sur l'ultrafiltration du lait avec des membranes minérales Ceraver ont montré que le conditionnement de celles-ci a une influence sur les débits de perméation (BENNASAR, 1984). Nous avons comparé dans les mêmes conditions les colmatages obtenus sur une membrane neuve, sèche (alumine non hydratée) ou mouillée par trempage de $5 \mathrm{~min}$ dans d'eau permutée (alumine hydratée).

Les résultats montrent d'une part que le colmatage se produit très rapidement dès les premières minutes de filtration (fig. 5a) et que d'autre part les taux 
TABLEAU I

Comparaison des teneurs en matières azotées totales (MAT), calcium (Ca) et phosphore $(P)$ des colmatages sur membrane sèche et mouillée, pour différents temps de contact avec le lait reconstitué

Total nitrogen matter (MAT), calcium (Ca) and phosphorus (P) quantity of fouling on dry and wet membrane, for different skimmilk contact times

\begin{tabular}{|c|c|c|c|c|c|c|c|c|c|}
\hline & \multicolumn{3}{|c|}{$\mathrm{MAT}^{*}, \mathrm{mg}$} & \multicolumn{3}{|c|}{$\mathrm{Ca}^{*}, \mathrm{mg}$} & \multicolumn{3}{|c|}{$\mathrm{P}^{*}, \mathrm{mg}$} \\
\hline & $30 \mathrm{~min}$ & $1 \mathrm{~h}$ & $2 \mathrm{~h}$ & $30 \mathrm{~min}$ & $1 \mathrm{~h}$ & $2 \mathrm{~h}$ & $30 \mathrm{~min}$ & $1 \mathrm{~h}$ & $2 \mathrm{~h}$ \\
\hline Sèche & 99 & 110 & 115 & 3,92 & - & 3,93 & 1,92 & 2,74 & 3,00 \\
\hline Mouillée & 53 & 61 & 75 & 2,60 & 2,80 & 3,15 & 1,15 & 1,55 & 2,09 \\
\hline
\end{tabular}

* Les teneurs sont rapportées à une surface membranaire identique $\left(33 \mathrm{~cm}^{2}\right)$.

* Quantity are given for à $33 \mathrm{~cm}^{2}$ membrane surface. 


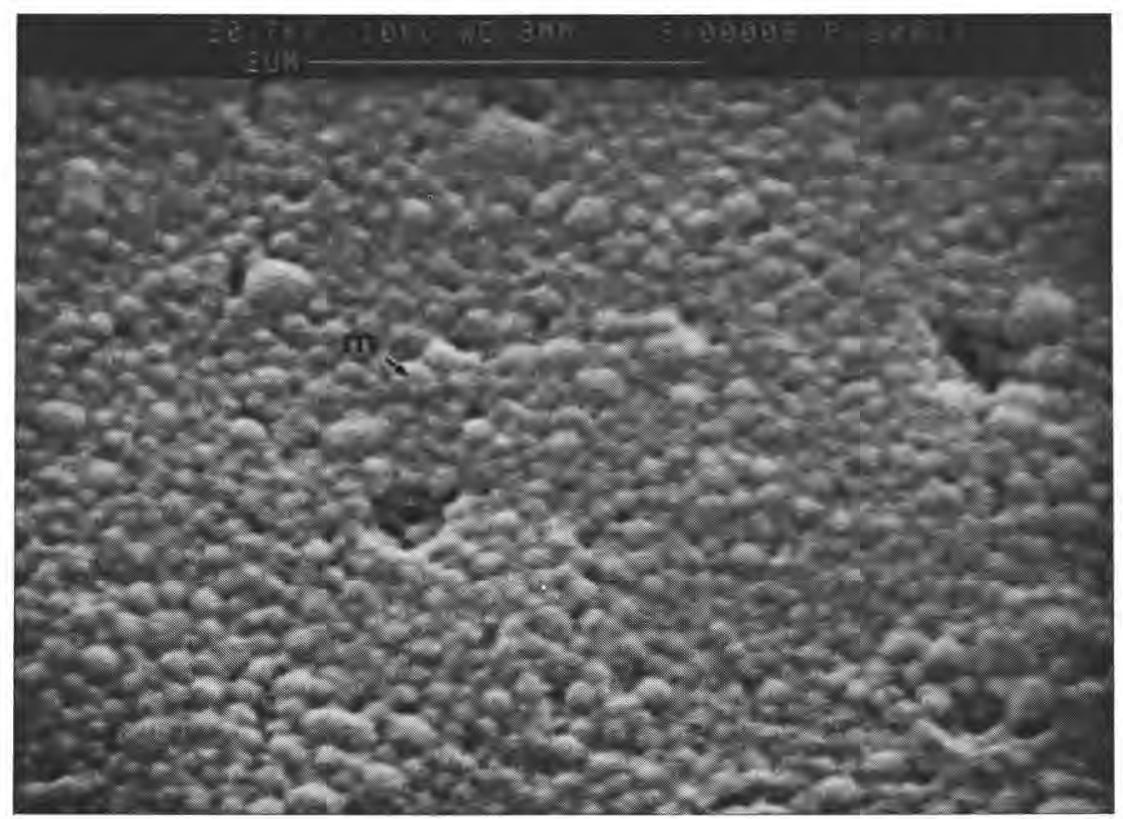

Fig. $4 \mathrm{c}$

Membrane colmatée après $30 \mathrm{~min}$ de contact avec le lait écrémé reconstitué (fixation c). Surface du dépôt avec une structure micellaire poreuse régulière ( $m=$ micelle) $(1000<$ Diamètre des micelles < 2000 A).

Membrane fouling after skimmilk contact time of 30 min (fixation c). Deposit surface with a regular porous micellar structure $(m=$ micelle $)(1000<$ micelle diameter $<2000$ A).

des principaux constituants (matières azotées totales, calcium et phosphore) du colmatage augmentent avec le temps et que ce colmatage est à chaque instant plus important avec la membrane sèche $(70 \%$ pour la MAT, $30 \%$ pour Ca, $60 \%$ pour P). Cette différence s'explique par l'importance plus grande des forces de capillarité en milieu poreux vide (membrane sèche) qui sont les principales responsables du transfert de matière du lait vers la membrane. Il est possible également que les liaisons avec l'alumine lors de l'adsorption des constituants du lait soient plus fortes lorsque celle-ci n'est pas hydratée et que cela conduise à un accroissement du dépôt. L'évolution différente selon les éléments reflète peutêtre également une variation de composition du colmatage.

\section{Influence du type de lait sur le colmatage en fonction du temps}

Dans ce cas, nous distinguons les teneurs des éléments (matières azotées totales, calcium et phosphore) dans les différentes solutions de récupération et la membrane (cf. $\S$ «Matériel et méthodes»). La solution de rinçage avec agitation (RAA) permet de récupérer le film liquide restant en surface du dépôt après élimination du lait, dont une partie correspond à la couche de polarisation. En 


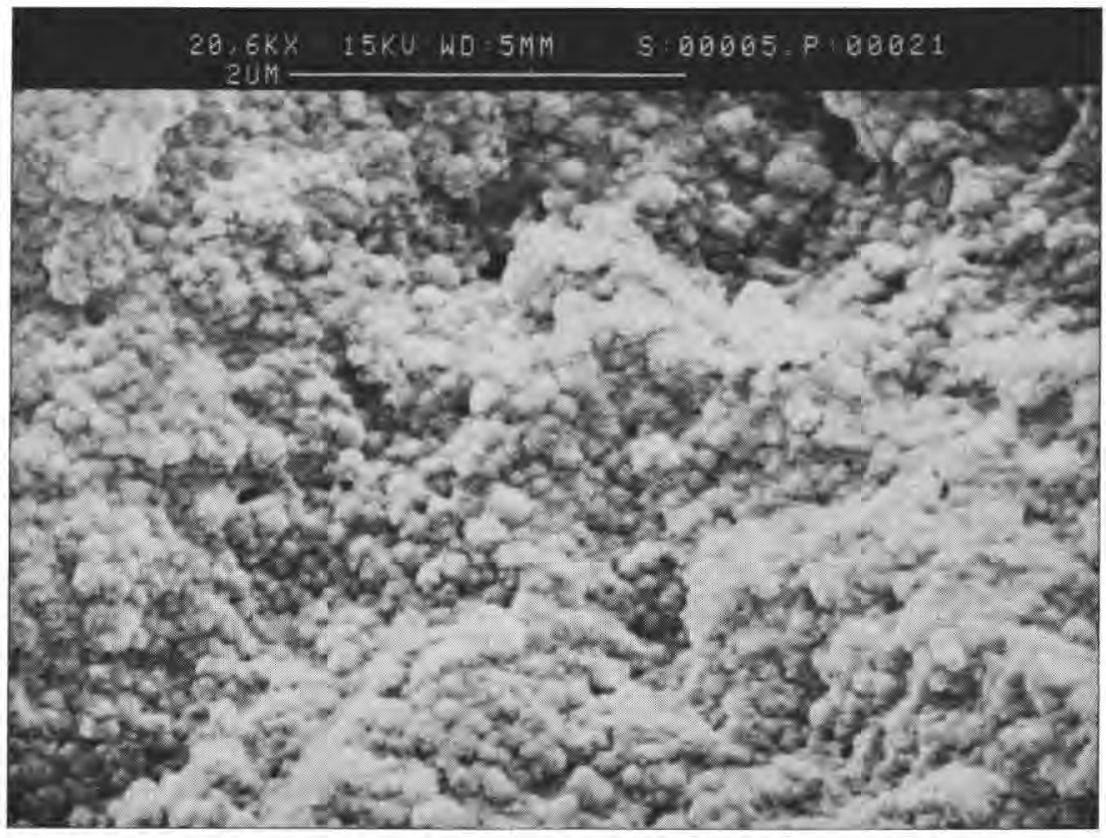

Fig. 4 d

Idem figure $14 \mathrm{c}$ : coupe du dépôt.

Idem figure $4 \mathrm{c}$ : section of deposit.

outre cette fraction contient également une partie des éléments minéraux du perméat présents dans le poreux; les résultats de son analyse ne peuvent pas être utilisés et sont seulement donnés à titre indicatif. La solution de récupération aux ultrasons (US) est constituée par la majeure partie du dépôt. Il subsiste toujours après passage aux ultrasons une faible quantité de matière dans la membrane qui correspond sans doute au colmatage initial par adsorption (AM). Les expériences ont été réalisées avec un lait cru entier et un lait cru écrémé.

\section{Lait cru entier (tabl. 2)}

Nous observons un colmatage élevé dont l'importance augmente en fonction du temps par suite de l'accroissement de la fraction US, c'est-à-dire du dépôt, qui représente au moins $80 \%$ du total protéique. Compte tenu des réserves émises ci-dessus concernant la fraction RAA, l'adsorption membranaire n'intervient que pour 3 à $5 \%$.

La valeur des rapports $\mathrm{Ca} / \mathrm{MAT}$ et P/MAT dans le dépôt (US) plus importante que dans les micelles de caséines, mais plus faible que dans le lait, et leur augmentation en fonction du temps résultent sans doute de l'augmentation de la teneur en $\mathrm{Ca}$ et $\mathrm{P}$ due à une diminution de la diffusion des sels vers la solution provoquée par la résistance du dépôt. Elles témoignent d'une participation importante du calcium et du phosphore dans le colmatage. Cet accroissement de la 
TABLEAU II

Teneurs en matières azotées totales (MAT), calcium $(C a)$ et phosphore $(P)$ des différentes fractions de récupération du colmatage (RAA, US, AM) en fonction du temps de contact avec le lait cru entier

Total nitrogen matter (MAT), calcium $(\mathrm{Ca})$ and phosphorus (P) quantity of fouling (RAA, US, AM) for different raw milk contact times

\begin{tabular}{|c|c|c|c|c|c|c|c|c|c|c|c|c|c|}
\hline \multirow{2}{*}{$\begin{array}{l}\text { Fractions } \\
\text { Temps }\end{array}$} & \multicolumn{4}{|c|}{$\mathrm{MAT}^{*}, \mathrm{mg}$} & \multicolumn{3}{|c|}{$\mathrm{Ca}^{*}, \mathrm{mg}$} & \multicolumn{3}{|c|}{$\mathrm{P}^{*}, \mathrm{mg}$} & \multirow{2}{*}{$\begin{array}{l}\mathrm{Ca} / \mathrm{MAT}^{* * *} \\
\quad \times 100\end{array}$} & \multirow{2}{*}{$\begin{array}{l}\mathrm{P} / \mathrm{MAT}^{* * *} \\
\quad \times 100\end{array}$} & \multirow{2}{*}{$\mathrm{Ca} / \mathrm{P}^{* *}$} \\
\hline & RAA & US & $\mathrm{AM}$ & Total & RAA & US & Total & RAA & US & Total & & & \\
\hline $30 \mathrm{~min}$ & 29 & 68 & 3 & 100 & 2,10 & 2,82 & 4,92 & 1,00 & 1,36 & 2,36 & 4,1 & 2,0 & 2,1 \\
\hline $2 \mathrm{~h}$ & 34 & 97 & 4 & 135 & 1,86 & 4,08 & 5,94 & 0,98 & 2,30 & 3,28 & 4,2 & 2,4 & 1,8 \\
\hline $5 \mathrm{~h}$ & 25 & 138 & 5 & 168 & 2,17 & 6,67 & 8,84 & 1,01 . & 3,66 & 4,67 & 4,8 & 2,7 & 1,8 \\
\hline
\end{tabular}

* Les teneurs sont rapportées à une surface membranaire identique $\left(33 \mathrm{~cm}^{2}\right)$.

** Les rapports $\mathrm{Ca} / \mathrm{MAT} /, \mathrm{P} / \mathrm{MAT}$ et $\mathrm{Ca} / \mathrm{P}$ sont calculés pour la fraction US (dépôt).

* Quantity are given for a $33 \mathrm{~cm}^{2}$ membrane surface.

$* * \mathrm{Ca} / \mathrm{MAT}, \mathrm{P} / \mathrm{MAT}$ and $\mathrm{Ca} / \mathrm{P}$ are given for US fraction (deposit). 


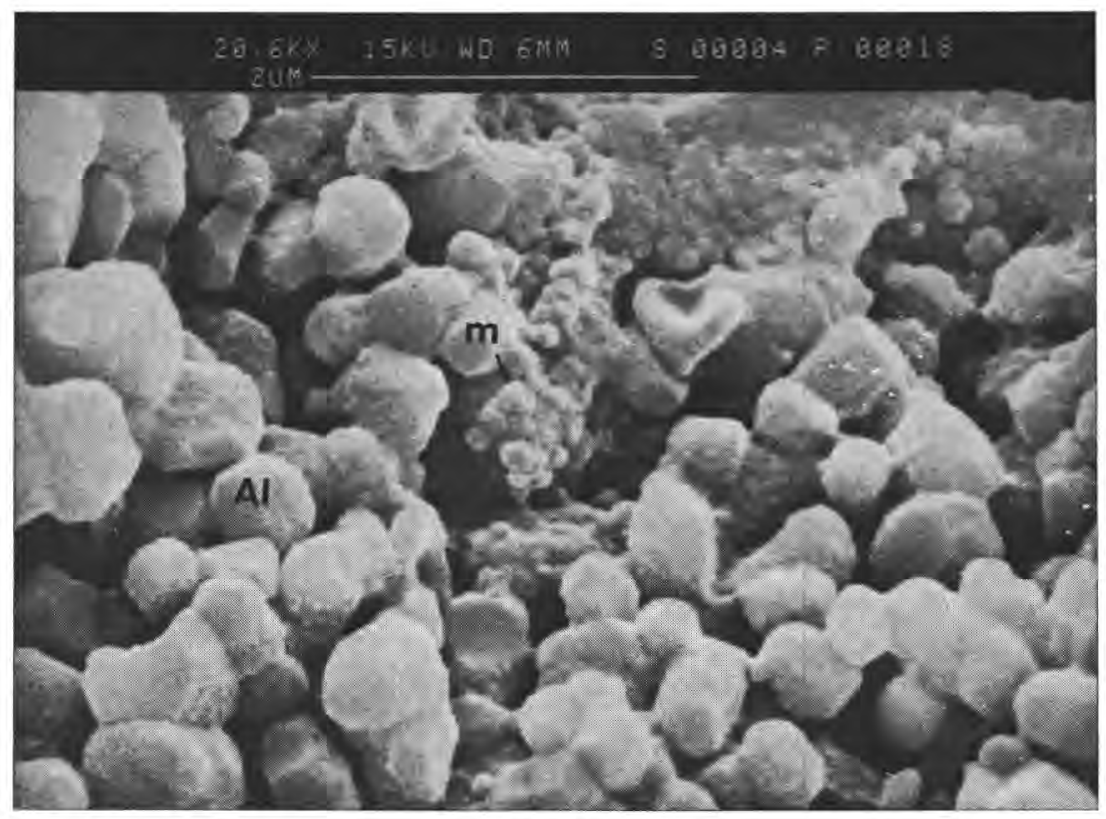

Fig. $4 \mathrm{e}$

Membrane colmatée après $30 \mathrm{~min}$ de contact avec le lait écrémé reconstitué (fixation c) : Interface membrane - dépôt : grains d'alumine enrobés $(A L)$, micelles de caséines ( $m$ ) incluses dans les grains d'alumine.

Membrane fouling after skimmilk contact time of $30 \mathrm{~min}$ (fixation c) : Membrane deposit interface : coated alumina grains $(A L)$, included casein micelles $(m)$ in alumina grains.

teneur en sels pouvant aboutir à la création de ponts salins entre les micelles de caséines comme l'ont indiqué différents auteurs dans le cas de l'osmose inverse (SKUDDER et al., 1977).

La tendance à la diminution du rapport $\mathrm{Ca} / \mathrm{P}$ en fonction du temps peut être le signe d'un accroissement de la taille des micelles ou d'une diminution relative des protéines du sérum dans le dépôt à mesure que l'on s'éloigne de la membrane.

Par ailleurs, la figure $5 \mathrm{~b}$ montrant la présence de vacuoles résiduelles provenant de la sublimation de la matière grasse lors des prétraitements des échantillons pour la microscopie électronique révèle la part importante prise par les globules gras dans la structure du dépôt.

\section{Lait cru écrémé (tabl. 3)}

Contrairement au lait entier, nous observons un colmatage plus faible, diminuant en fonction du temps, surtout entre $30 \mathrm{~min}$ et $2 \mathrm{~h}$; cette diminution est essentiellement due dans ce cas aussi à la baisse de la fraction US, c'est-à-dire du dépôt.

La répartition des différentes fractions protéiques du colmatage est ici également en faveur du dépôt (au moins $70 \%$ ) avec un pourcentage toujours très 


\section{TABLEAU III}

Teneurs en matières azotées totales $(M A T)$, calcium $(\mathrm{Ca})$ et phosphore $(P)$ des différentes fractions de récupération du colmatage (RAA, US, AM) en fonction du temps de contact avec le lait cru écrémé

Total nitrogen matter (MAT), calcium (Ca) and pphosphorus (P) quantity of fouling (RAA, US, AM) for different raw skimmilk contact times

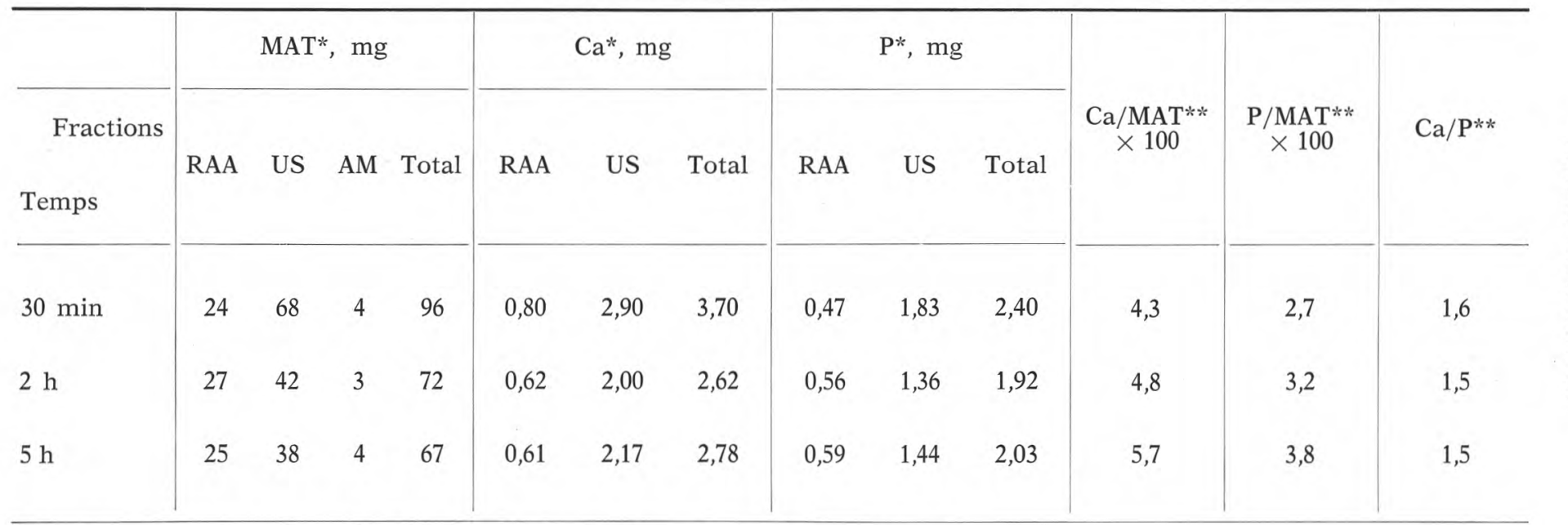

* Les teneurs sont rapportées à une surface membranaire identique $\left(33 \mathrm{~cm}^{2}\right)$.

** Les rapports $\mathrm{Ca} / \mathrm{MAT}, \mathrm{P} / \mathrm{MAT}$ et $\mathrm{Ca} / \mathrm{P}$ sont calculés pour la fraction US (dépôt).

* Quantity are given for a $33 \mathrm{~cm}^{2}$ membrane surface.

** $\mathrm{Ca} / \mathrm{MAT}, \mathrm{P} / \mathrm{MAT}$ and $\mathrm{Ca} / \mathrm{P}$ are given for US fraction (deposit). 


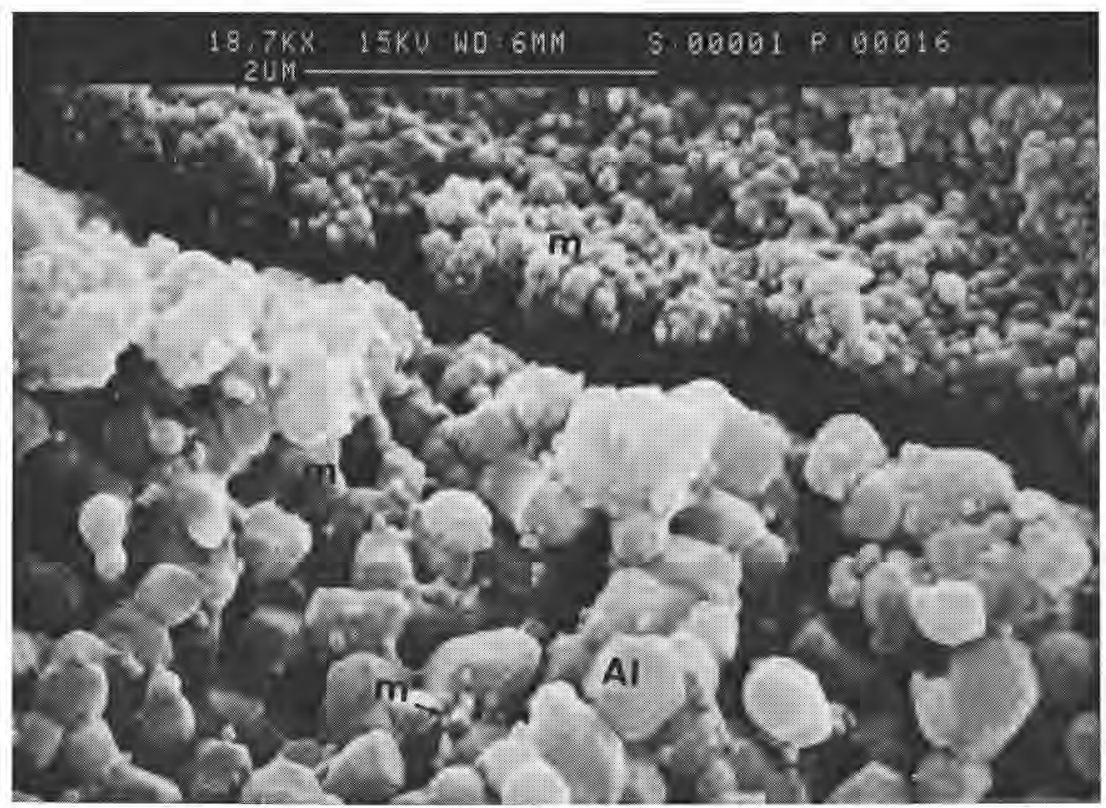

Fig. 5 a

Membrane colmatée après 5 min de contact avec le lait écrémé reconstitué (fixation c). Structure micellaire du dépôt (d) et colmatage interne avec adsorption de micelles (m) sur les grains d'alumine (AL).

Membrane fouling after skimmilk contact time of $5 \mathrm{~min}$ (fixation c). Micellar structure of deposit (d) and internal fouling with micelles absorbancy $(m)$ on the alumina grains $(A L)$.

faible ( 4 à $6 \%$ ) de constituants adsorbés. La différence de comportement suivant le type de lait quant à l'évolution du colmatage en fonction du temps s'explique peut-être par l'absence ou la présence des globules gras dans le dépôt. Leur présence limiterait les phénomènes de rediffusion des constituants du dépôt vers le lait sous l'effet du gradient de concentration et donc la diminution du dépôt au cours du temps puisque par ailleurs la mesure des volumes de lait versé montre que les transferts de matière à travers la membrane sont aussi importants avec un lait entier qu'avec un lait écrémé.

Par ailleurs, les rapports $\mathrm{Ca} / \mathrm{MAT}$ et $\mathrm{P} / \mathrm{MAT}$ sont plus élevés et augmentent plus vite en fonction du temps que dans le cas précédent. Les résultats sont sans doute en relation avec la compacité du colmatage due à l'absence de globules gras; le dépôt serait alors constitué par un réseau dense de micelles reliées par des ponts salins nombreux limitant la diffusion des ions $\mathrm{Ca}$ et $\mathrm{P}$.

Le rapport $\mathrm{Ca} / \mathrm{P}$ plus faible dans le cas du lait écrémé est difficilement explicable actuellement en dehors d'une participation différente de ces ions dans la structure du dépôt.

\section{Analyse du dépôt de colmatage par électrophorèse}

L'électrophorèse est réalisée sur la première solution de récupération aux ultrasons (US) représentative d'une partie du dépôt obtenu par contact de la 


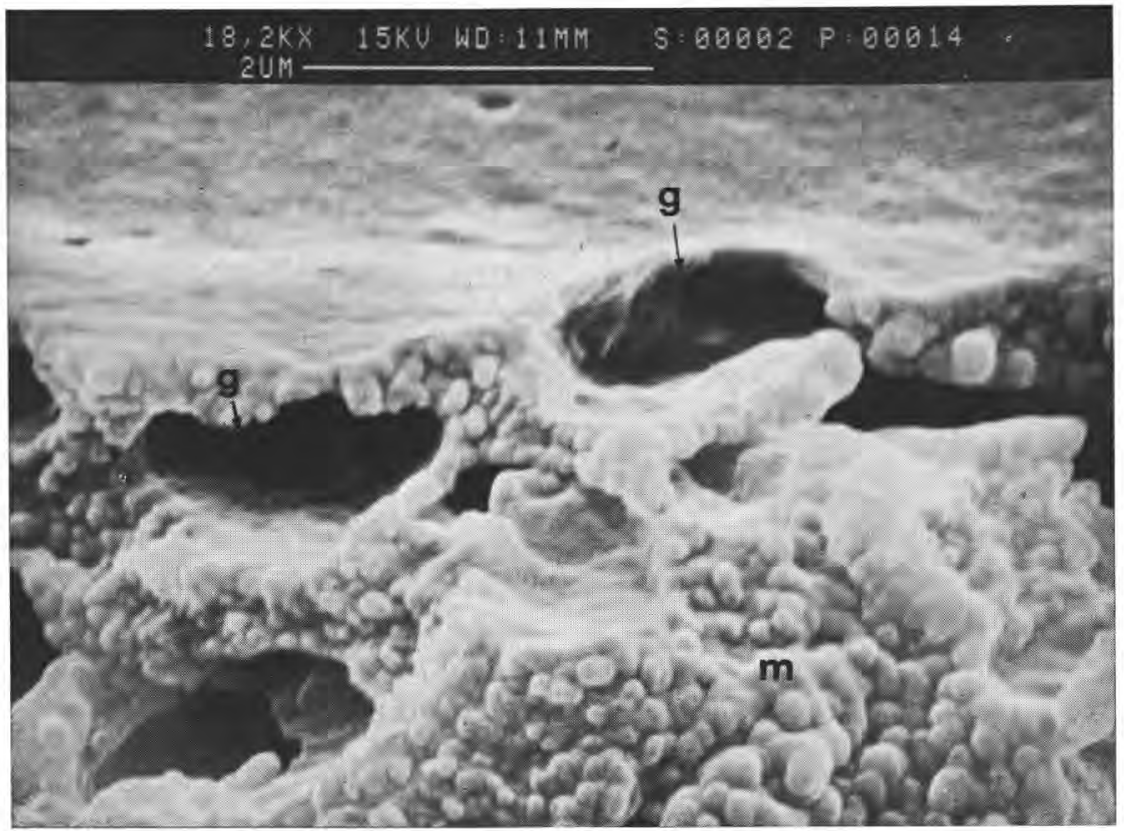

Fig. $5 \mathrm{~b}$

Membrane colmatée après $2 \mathrm{~h}$ de contact avec le lait cru entier (fixation b). Vacuoles laissées par les globules gras $(\mathrm{g})$ dans la matrice protéique des micelles $(\mathrm{m})$.

Membrane fouling after raw milk contact time of 2 hours (fixation b). Fat globule impression $(\mathrm{g})$ in micellar deposit $(\mathrm{m})$.

\section{TABLEAU IV}

Comparaison de la répartition des principaux constituants protéiques du dépôt (US) et du lait normal (LN)

Comparison of relative quantity of major proteins in the deposit (US) and in the milk (LN)

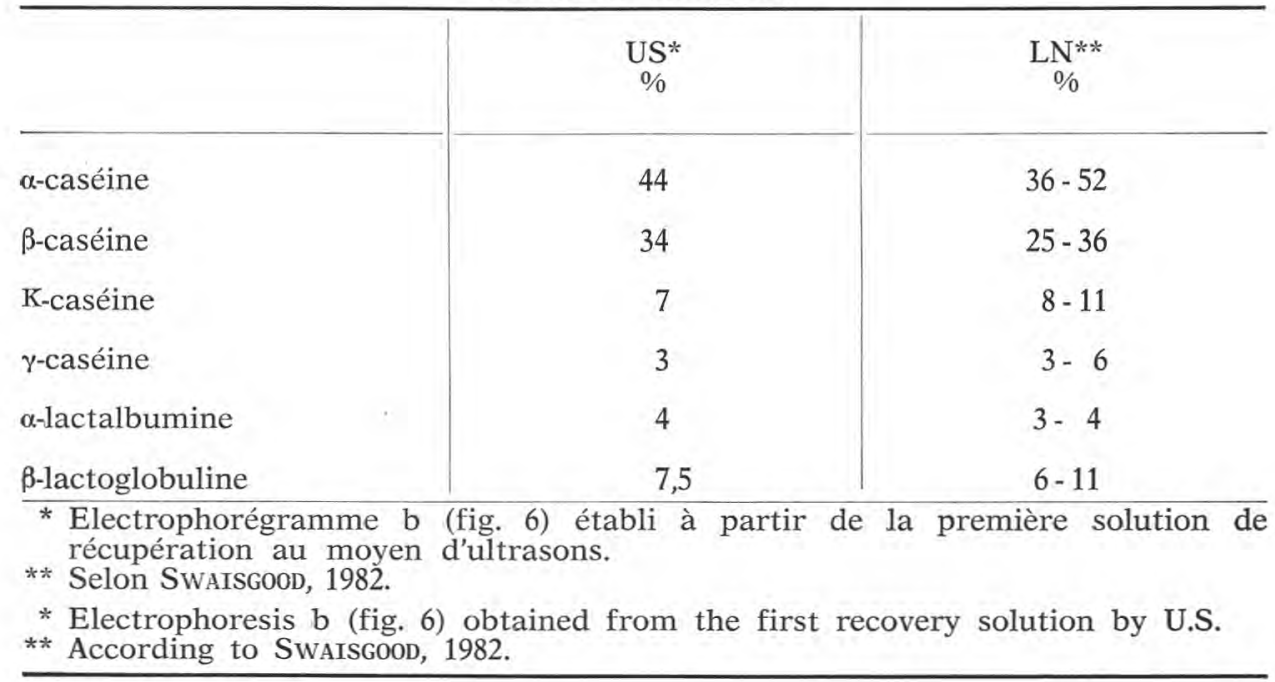




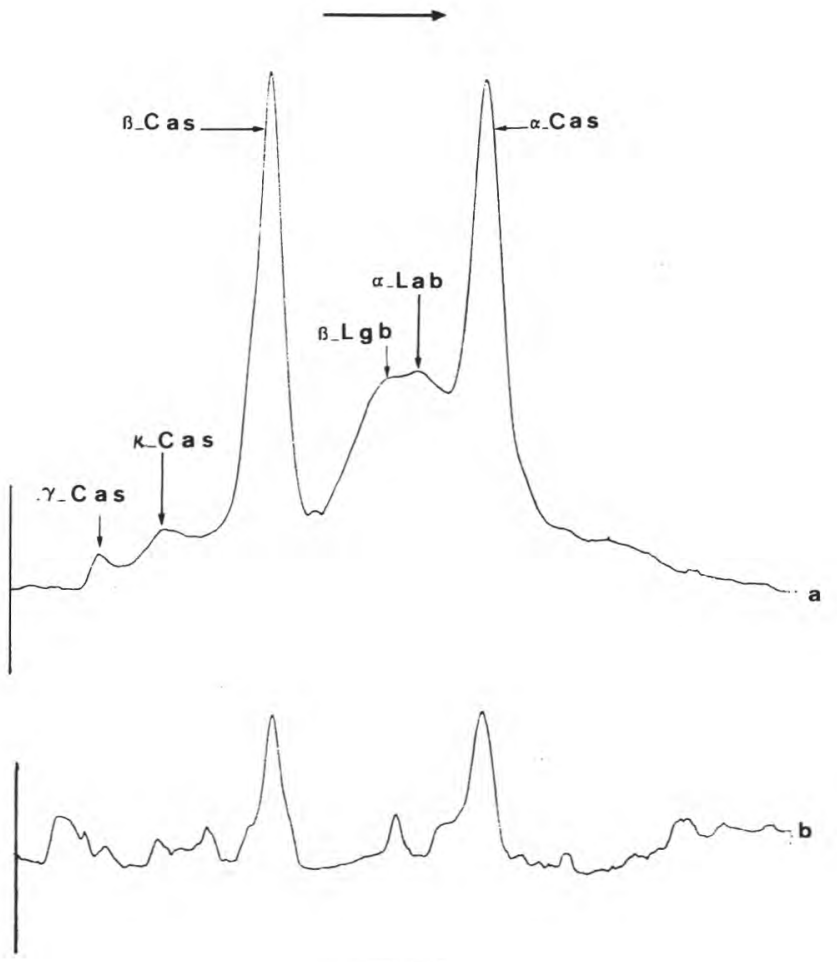

Fig. 6

Enregistrement au scanner de bandes d'électrophorèse

Fig. 6 a : Electrophorégramme d'un mélange de solutions étalons de protéines du lait : $\alpha$-lactalbumine, $\beta$-lactoglobuline et caséine isoélectrique.

Fig. 6 b : Electrophorégramme de la fraction US du dépôt obtenu avec le lait cru entier.

Scanning of polyacrylamide gel electrophoresis

Fig. $6 a$ : Protein standart mixture : $\alpha$-lactalbumin, $\beta$-lactoglobulin and casein.

Fig. $6 b$ :US - fraction of raw milk deposit.

membrane pendant $5 \mathrm{~h}$ avec du lait cru entier par comparaison avec une solution étalon contenant les différentes caséines $(\alpha, \beta, \chi$ et $\gamma$ ), la $\beta$-lactoglobuline et l' $\alpha$-lactalbumine. Les électrophorégrammes correspondants sont représentés sur la figure 6. Nous retrouvons la présence de tous les constituants protéiques initiaux du lait comme Glover et Brooker (1974) et SKUdDer et al. (1977) l'ont également montré lors de l'étude électrophorétique du dépôt obtenu lors du traitement par osmose inverse du lait. En outre la répartition des principaux constituants protéiques dans le dépôt est voisine de celle du lait normal comme le montrent les valeurs du tableau 4. 


\section{Conclusion}

La technique de microscopie à balayage nous a permis de visualiser le phénomène de colmatage obtenu en statique et de confirmer certaines des hypothèses proposées pour son mécanisme. C'est ainsi que nous avons pu mettre en évidence :

- la formation très rapide du colmatage ;

- l'adsorption initiale en faible quantité de constituants non micellaires sur les grains d'alumine composant la membrane ;

- la pénétration réduite du colmatage dans les premières couches des grains d'alumine membranaires ;

- l'importance quantitative du dépôt (60 à $80 \%$ ) par rapport aux autres fractions du colmatage ;

- la structure poreuse du dépôt constitué par l'agglomération des micelles de caséines ;

- l'incorporation des globules gras dans la matrice protéique du dépôt;

- l'évolution différente de l'épaisseur du dépôt avec le temps de contact suivant que le lait est entier (augmentation) ou écrémé (diminution).

Les analyses biochimiques et électrophorétiques du colmatage nous ont permis de confirmer sa composition protéique avec présence de tous les constituants d'origine du lait (caséines $\alpha, \beta$, $\chi$ et $\gamma ; \beta$-lactoglobuline et $\alpha$-lactalbumine), la participation importante de sels tels que le calcium et le phosphore qui interviennent sans doute dans la constitution du réseau et l'évolution du dépôt en fonction du temps.

Rappelons que nos résultats ont été obtenus en statique avec une membrane de microfiltration à structure granulaire permettant une séparation de type ultrafiltration lors de son utilisation pour le traitement du lait (BENNASAR, 1984). Il est probable qu'une telle étude réalisée avec des membranes polymères d'ultrafiltration à structure non granulaire et de diamètre moyen de pores beaucoup plus faible conduirait à des observations différentes, en particulier en ce qui concerne le colmatage interne.

Cependant le bilan de nos résultats confirme l'intérêt du couplage de ces techniques pour la compréhension du phénomène de colmatage, lesquelles devraient pouvoir s'appliquer avec succès également aux membranes de deuxième génération.

Enfin nous envisageons de poursuivre cette étude en dynamique, ce qui permettra de connaître le rôle exact du colmatage lors de la filtration tangentielle sur membrane.

Reçu le 19-8-1985.

Accepté pour publication le 22-2-1986

\section{Bibliographie}

Baklouti S., Aimar P., Sanchez V., 1984. Adsorption et transferts de matière en ultrafiltration. Lait, 64, 638-639, 217-238.

BenNaSAR M., 1984. Etude de l'ultrafiltration sur membranes minérales. Application au lait. Thèse de Docteur d'Etat, Montpellier.

Carroll R.J., Thompson M.P., Nutting G.C., 1968. Glutaraldehyde fixation of casein micelles for Electron Microscopy. J. Dairy Sci, 51, 12, 1903-1908. 
GERNEDEL C., 1980. Uber die Ultrafiltration von milch und die den Widerstand der Ablagerungsschicht beeinflussenden Faktoren. Thèse de Docteur-ingénieur, Munich.

GLover F.A., BRooKer B.E., 1974. The structure of the deposit formed on the membrane during the concentration of milk by reverse osmosis. J. Dairy Res., 41, 1, 89-93.

KALAB M., 1981. Electron Microscopy of milk products : a review of techniques. Scanning Electron Microsc., III, 453-472.

Kalab M., Phipps-Todd B.E., Allan-Wojtas P., 1982. Milk gel structure : XIII. Rotary shadowing of casein micelles for electron microscopy. Milchwissenschaft, 37 (9), 513-518.

KALAB M., 1984. Artefacts in conventional scanning electron microscopy of some milk products. Food Microstruc., 3, 95-111.

Lee D.N., Miranda M.G., Merson R.L., 1975. - Scanning Electron Microscope studies of membrane deposits from whey ultrafiltration. J. Food Technol., 10, 139-146.

PIEN J., 1969. Dosage du phosphore dans le lait. Lait, 49 (483-484), 175-188.

PoIRIER D., 1985. Contribution à l'étude de la microfiltration tangentielle sur membranes minérales et de son application à la clarification, et la stabilisation des vins. Thèse de Docteur-ingénieur, Montpellier.

PoRTER M.C., 1972. Concentration Polarization with membrane ultrafiltration. Ind. Eng. chem. Prod. Res. Develop., 11 (3), 234-248.

ŚKUdDER P.J., GLOVER F.A., GREEN M.L., 1977. An examination of factors affecting the reverse osmosis of milk with special reference to deposit formation. J. Dairy Res., 44, 2, 293-307.

SwaIsGood H.E., 1982. Chemistry of milk protein. In : Developments in dairy chemistry. 1 Ed. by Fox P.F., Applied Science Publishers, London and N.Y., 1-59. 